\title{
MÉTODOS DE CONTROLE DE PLANTAS INVASORAS NA CULTURA DO CAFEEIRO (Coffea arabica L.) E COMPONENTES DA ACIDEZ DO SOLO ${ }^{(1)}$
}

\author{
Elifas Nunes Alcântara ${ }^{(2)}$, Júlio César Azevedo Nóbrega ${ }^{(3)}$ \& Mozart \\ Martins Ferreira $^{(4)}$
}

\begin{abstract}
RESUMO
Em lavouras perenes, como na cultura do cafeeiro, o controle de plantas invasoras tem sido feito por meio de métodos manuais, mecanizados, químicos e associações destes. De modo geral, têm-se avaliado os diferentes métodos sob o ponto de vista de eficiência e de custo no controle das plantas invasoras; no entanto, a influência deles sobre as condições químicas do solo, praticamente, não tem sido estudada, principalmente a longo prazo. $O$ objetivo deste estudo foi avaliar o efeito de diferentes métodos de controle de plantas invasoras na cultura do cafeeiro sobre os componentes da acidez de um Latossolo Vermelho distroférrico da região de São Sebastião do Paraíso, MG. Sete tratamentos de controle de plantas invasoras foram avaliados: roçadora (RÇ), grade (GR), enxada rotativa (RT), herbicida de pós-emergência (HC), herbicida de pré-emergência (HR), capina manual (CM) e testemunha sem capina (SC), dispostos em blocos casualizados com três repetições. Amostras de solo, em cada tratamento, foram coletadas a cada dois anos, a partir de 1980, nas camadas de 0-0,15 e 0,15-0,30 m, para avaliação de $\mathrm{pH}, \mathrm{Al}^{3+}$, acidez potencial $(\mathrm{H}+\mathrm{Al})$ e saturação por $\mathrm{Al}^{3+}(\mathrm{m})$. O sistema $\mathrm{HR}$ aumentou o teor $\mathrm{e}$ a saturação por $\mathrm{Al}^{3+}$ e a acidez potencial e diminuiu o $\mathrm{pH}$, quando comparado com os demais métodos de controle de plantas invasoras, principalmente com a testemunha (SC). O tratamento SC mostrou efeito contrário ao do HR, aumentando
\end{abstract}

\footnotetext{
(1) Parte da Tese de Doutorado do primeiro autor apresentada ao Departamento de Fitotecnia da Universidade Federal de Lavras - UFLA. Recebido para publicação em outubro de 2005 e aprovado em julho de 2007.

${ }^{(2)}$ Pesquisador da Empresa de Pesquisa Agropecuária de Minas Gerais - EPAMIG/CTSM. Campus Universitário da Universidade Federal de Lavras - UFLA. Caixa Postal 176, CEP 37200-000 Lavras (MG). Bolsista da FAPEMIG. E-mail: elifas@epamig.ufla.br

${ }^{(3)}$ Professor do Centro de Ciências Agrárias, Universidade Federal do Piauí - UFPI. Campos da Socopo, CEP 64049-550 Teresina (PI). E-mail: jnobrega@ufpi.br

${ }^{(4)}$ Professor do Departamento de Ciência do Solo, UFLA. E-mail: mozartmf@ufla.br
} 
os valores de pH e diminuindo o teor de $\mathrm{Al}^{3+}$ e a saturação por $\mathrm{Al}^{3+}$, em ambas as camadas de solo. O RÇ foi o tratamento que mais se aproximou do SC, e os demais tratamentos, no geral, não apresentaram comportamento diferenciado.

Termos de indexação: manejo de invasoras, qualidade química do solo.

\title{
SUMMARY: WEED CONTROL METHODS AND SOIL ACIDITY COMPONENTS IN COFFEE PLANTATION (Coffea arabica $L$.)
}

\begin{abstract}
In perennial agriculture, such as coffee plantation, weeds are controlled by hand, mechanized, and chemical weeding and their combinations. Methods that differ in terms of efficiency and costs have been evaluated; however, the influence of these methods on the soil chemical conditions has not been studied, particularly in the long term. This study aimed to evaluate the effect of different weed control methods on soil acidity components of a coffee plantation on a dystroferric Red Latosol in an experiment installed at EPAMIG Experimental Station, in São Sebastião do Paraíso, MG, Brazil. Seven weed control treatments were evaluated: mower $(M W)$, coffee tandem disk harrow (TD), rotary tiller $(\mathrm{RT})$, post $(\mathrm{CH})$ and pre-emergency herbicide $(\mathrm{RH})$ application, hand hoe $(\mathrm{HH})$ and no weed control (NC). The treatments were evaluated in a randomized block design with three repetitions. Soil samples were collected every two years, for each treatment, starting in 1980 , in the $0-0.15$ to $0.15-0.30 \mathrm{~m}$ soil layers and assessed for $\mathrm{pH}, \mathrm{Al}^{3+}\left(\mathrm{mmol}_{\mathrm{c}} \mathrm{dm}^{-3}\right)$, effective acidity $(H+A l)\left(\mathrm{mmol}_{c} \mathrm{dm}^{-3}\right)$, and $A l^{3+}$ saturation (\%). The $H R$ system increased $\mathrm{Al}^{3+}$ content and saturation and the effective acidity and reduced the $\mathrm{pH}$, compared to the other weed control methods, especially the control (NC). Contrarily to the $R H$, the NC treatment resulted in higher $\mathrm{pH}$ values, $\mathrm{Al}^{3+}$ content and $\mathrm{Al}^{3+}$ saturation in both soil layers. The MW treatment was closest to the SC treatment, while the other weed control systems did not differ significantly.
\end{abstract}

Index terms: weed management, chemical soil quality.

\section{INTRODUÇÃO}

A conversão de ecossistemas nativos em ecossistemas agrícolas resulta, quase sempre, na perda de matéria orgânica do solo, a qual está intimamente associada à sua qualidade. Isso ocorre devido à estreita relação existente entre o teor de matéria orgânica e a magnitude de várias propriedades químicas, físicas e biológicas do solo (Bayer \& Mielniczuk, 1997; Bayer \& Bertol, 1999).

A rápida degradação de alguns atributos químicos do solo quando sob cultivo intensivo tem requerido a aplicação contínua de corretivos e de fertilizantes ao solo, fato que contribui para reduzir a sustentabilidade econômica dos ecossistemas agrícolas, principalmente em países de clima tropical em desenvolvimento, devido ao alto custo desses insumos. No geral, os solos de regiões tropicais são ácidos e apresentam elevados teores de $\mathrm{Al}$ trocável.

O monitoramento de atributos físicos, químicos e biológicos do solo, com vista a sugerir modificações nos sistemas de manejo utilizados na produção agrícola, tem sido prática comum. Diversas técnicas de manejo influenciam o teor de matéria orgânica do solo e, conseqüentemente, os seus atributos químicos. $\mathrm{O}$ efeito de técnicas de cultivo que permitem proteção da superfície do solo, pelo retorno dos resíduos das culturas e das plantas invasoras, como ocorre no sistema plantio direto, tem-se mostrado positivo em termos de melhoria de propriedades químicas do solo (Reicosky et al., 1995; Silva \& Ribeiro, 1995; Iyamuremye \& Dick, 1996; Santos \& Tomm, 1996; Bayer \& Mielniczuk, 1997), devido, principalmente, à melhoria do teor de matéria orgânica (Aase \& Pikul Jr., 1995; Santos \& Tomm, 1996; Bayer \& Mielniczuk, 1997).

A recuperação do potencial produtivo do solo, pelo uso de plantas, como gramíneas e leguminosas (Santos et al., 2001), e a utilização de sistemas de sucessão e de rotação de culturas (Bayer \& Mielniczuk, 1997; Bayer \& Bertol, 1999), também tem sido verificada, devido ao aumento que promovem no teor de matéria orgânica e, conseqüentemente, na melhoria de condições químicas do solo. Relação linear entre o aporte de resíduos vegetais durante 11 anos, em diferentes sistemas de culturas, e o teor de $\mathrm{C}$ do solo, em sistema plantio direto, foi observada por Burle et al. (1997). 
Em lavouras perenes, como a cultura do cafeeiro (Coffea arabica), o manejo do solo se resume, basicamente, na adição de corretivos/fertilizantes e no controle de plantas invasoras. O controle de plantas invasoras tem sido feito por meio de métodos manuais, mecanizados, químicos e associações destes. De modo geral, tem-se buscado avaliar diferentes métodos sob o ponto de vista de eficiência e de custo no controle dessas plantas invasoras. Todavia, a influência de diferentes métodos sobre as condições químicas do solo, praticamente, não tem sido estudada, sobretudo a longo prazo. O controle de plantas invasoras, quando adequadamente, feito, pode contribuir para melhoria da qualidade do solo, principalmente em decorrência da elevação do teor de matéria orgânica do solo promovida pela diversidade de espécies de plantas invasoras presentes na cultura (Alcântara \& Ferreira, 2000).

Diante do exposto, este estudo teve por objetivo avaliar o efeito de diferentes métodos de controle de plantas invasoras na cultura do cafeeiro sobre os componentes da acidez de um Latossolo Vermelho distroférrico.

\section{MATERIAL E MÉTODOS}

O estudo foi realizado na Fazenda Experimental da EPAMIG, em São Sebastião do Paraíso, MG ( $46^{\circ} 55^{\prime} \mathrm{W}$ e $\left.20^{\circ} 55^{\prime} \mathrm{S}\right)$, em um Latossolo Vermelho distroférrico textura argilosa (520 $\mathrm{g} \mathrm{kg}^{-1} \mathrm{de}$ argila, $360 \mathrm{~g} \mathrm{~kg}^{-1}$ de areia e $120 \mathrm{~g} \mathrm{~kg}^{-1}$ de silte), com $8 \%$ de declividade. A vegetação original da área de estudo era formada por floresta estacional semidecidual, transicional para Cerrado. A precipitação pluvial média anual da região é de $1.470,4 \mathrm{~mm}$ e a temperatura média anual de $20,8^{\circ} \mathrm{C}$, com média máxima e mínima de 27,6 e $14,1^{\circ} \mathrm{C}$, respectivamente.

\section{Condução do experimento, tratamentos e delineamento experimental}

O plantio de café foi realizado em 1974, empregando-se o cultivar Catuaí Vermelho LCH 2077 2-5-99, no espaçamento $4 \times 1 \mathrm{~m}$, totalizando 2.268 covas ha ${ }^{-1}$. Em 1977, deu-se a instalação do experimento, que continua sendo conduzido até os dias atuais. No entanto, o período contemplado neste estudo compreende as avaliações feitas entre os anos de 1980 a 1995 . A aplicação de fertilizantes no solo foi efetuada, desde a implantação, de acordo com as análises de solo e as recomendações técnicas para a cultura, elaboradas pela Comissão de Fertilidade do Solo do Estado de Minas Gerais (1989). A calagem foi feita desde a implantação da lavoura na área de projeção da copa das plantas.

No controle de plantas invasoras foram utilizados sete métodos, que constituíram os tratamentos, aplicados na parte central das entrelinhas de plantio,

também denominadas "ruas" (Quadro 1). As laterais das linhas de plantio, correspondendo às projeções das copas dos cafeeiros ou "saias", foram mantidas sempre limpas pelo uso de herbicidas e, ou, capinas manuais.

Todas as operações de controle foram efetuadas sempre que se observava $90 \%$ da "rua" coberta pelas plantas invasoras e, ou, estas apresentavam cerca de $0,45 \mathrm{~m}$ de altura. Assim, o número médio de operações necessárias para controle das plantas invasoras, durante cada ano, variou conforme o método (Quadro 1).

Para controle de plantas invasoras na condição de pré-emergência (HR), utilizou-se a mistura formulada dos herbicidas ametryn (2-etilamino-4-isopropilamino6-metiltio-s-triazina) + simazine (2-cloro-4-6-bisetilamino-S-triazina), nas doses de $2,4 \mathrm{~kg} \mathrm{ha}^{-1}$ de i.a., na formulação pó molhável, e de $2,25 \mathrm{~kg} \mathrm{ha}^{-1}$ de i.a., na formulação líquida, aplicadas com volume de calda de $400 \mathrm{~L} \mathrm{ha}^{-1}$. Quando da ocorrência de escapes, emergência de algumas plantas invasoras, estas eram controladas pela catação, com enxada manual, antes da aplicação do herbicida de pré-emergência.

Para controle em pós-emergência (HC) foi utilizada, inicialmente, uma mistura de paraquat [1,1'- dimetil4,4 ' bipiridilio íon (dicloreto)] e diquat $(6,7$ dihydrodipyrido [1,2-a-2'-1'-e] pyrazinediium, na proporção de $0,40 \mathrm{~kg} \mathrm{ha}^{-1}$ de i.a. Posteriormente, o controle foi efetuado pela aplicação de glyphosate N(fosfonometil) glicina, na dose de $1,44 \mathrm{~kg} \mathrm{ha}^{-1}$ de i.a., alternado com a mistura de glyphosate $+2,4-\mathrm{D}$ [ácido 2,4 diclorofenoxiacético], na proporção, respectivamente, de $160+120 \mathrm{~g} \mathrm{ha}^{-1}$ e dose de $640 \mathrm{~g}+480 \mathrm{~g} \mathrm{ha}^{-1}$ de i.a.

$\mathrm{O}$ delineamento experimental utilizado foi o de blocos casualizados, com sete tratamentos em três repetições. Cada parcela, constituída por três "ruas", era constituída por 108 plantas de café.

Quadro 1. Métodos de controle de plantas invasoras utilizados na cultura do cafeeiro em um Latossolo Vermelho distroférrico e os respectivos números de operações realizadas por ano

Tratamento

Roçadora (RÇ)

Grade (GR)

Enxada rotativa (RT)

Herbicida de pós-emergência (HC)

Herbicida de pré-emergência (HR)

Capina manual (CM)

Testemunha sem capina (SC)
Operação ano-1

\section{5}

3

3

3

2

5 


\section{Amostragem de solo e análises químicas}

As amostras de solo para as análises químicas foram coletadas a cada dois anos, a partir de 1982, coincidindo com os anos de baixa produção, após a "esparrama de cisco" (redistribuição da serapilheira). As amostras foram coletadas na "rua" central de cada tratamento, nas camadas de $0-0,15$ e $0,15-0,30 \mathrm{~m}$, em número de seis por camada e por parcela.

Foram avaliados o pH em água $(1: 2,5)$, acidez potencial $(\mathrm{H}+\mathrm{Al}), \mathrm{Al}$ trocável e saturação por $\mathrm{Al}^{3+}$, segundo Vettori (1969) e Embrapa (1979).

\section{Análises estatísticas}

Os resultados foram submetidos à análise de variância e de regressão. As análises de variância foram realizadas segundo o delineamento experimental de blocos ao acaso, em esquema de parcelas (métodos de controle de plantas invasoras) subdivididas no tempo (biênios), sendo as médias comparadas pelo teste de Duncan a $5 \%$. As equações de regressão foram determinadas para avaliar e descrever as respostas de todas as variáveis estudadas em função dos teores de matéria orgânica, em cada condição de controle de plantas invasoras. Além disso, foram determinados os coeficientes de correlação entre os componentes da acidez do solo, com os teores de matéria orgânica, com o objetivo de avaliar o grau de associação entre essas variáveis.

\section{RESULTADOS E DISCUSSÃO}

Acidez do solo e seus componentes ( $\mathrm{pH}$, $\mathbf{H}+\mathbf{A l}, \mathbf{A l}^{3+}$ e saturação por $\mathbf{A l}^{3+}$ )

Os tratamentos de controle de plantas invasoras influenciaram, significativamente, os componentes da acidez do solo: $\mathrm{pH}, \mathrm{H}+\mathrm{Al}$ e saturação por $\mathrm{Al}^{3+}$, nas camadas de $0-0,15 \mathrm{~m}(\mathrm{p}<0,05)$ e de $0,15-0,30 \mathrm{~m}$ $(\mathrm{p}<0,01), \mathrm{e} \mathrm{Al}^{3+}(\mathrm{p}<0,01)$.

O valor do $\mathrm{pH}$ do solo, ao longo do tempo de avaliação (biênios), nas camadas de $0-0,15$ e $0,15-0,30 \mathrm{~m}$ (Quadro 2), manteve-se, praticamente, inalterado. Entretanto, diferenças foram observadas quando se

Quadro 2. Valor de pH em água das camadas de 0-0,15 e 0,15-0,30 m de um Latossolo Vermelho distroférrico, em função do tratamento de controle de plantas invasoras na cultura do cafeeiro

\begin{tabular}{|c|c|c|c|c|c|c|c|c|c|}
\hline \multirow{2}{*}{ Tratamento } & \multicolumn{9}{|c|}{ Ano } \\
\hline & 1980 & 1982 & 1984 & 1986 & 1988 & 1991 & 1993 & 1995 & Média \\
\hline & \multicolumn{9}{|c|}{$0-0,15 \mathrm{~m}$} \\
\hline $\mathrm{RÇ}$ & 5,5 aA & $6,1 \mathrm{abA}$ & $6,0 \mathrm{abA}$ & 5,9 abA & $5,2 \mathrm{aA}$ & 6,6 aA & 5,8 aA & $6,0 \mathrm{bA}$ & $5,9 \mathrm{~b}$ \\
\hline GR & $5,2 \mathrm{aA}$ & $6,0 \mathrm{abA}$ & 5,8 abcA & $5,6 \mathrm{bA}$ & $5,2 \mathrm{aA}$ & $6,3 \mathrm{aA}$ & 5,7 aA & $6,2 \mathrm{bA}$ & $5,8 \mathrm{bc}$ \\
\hline $\mathrm{RT}$ & $5,4 \mathrm{aA}$ & $5,8 \mathrm{abA}$ & $5,5 \mathrm{bcA}$ & 5,9 abA & $5,1 \mathrm{aA}$ & $6,3 \mathrm{aA}$ & 5,5 aA & $6,1 \mathrm{bA}$ & $5,7 \mathrm{c}$ \\
\hline $\mathrm{HC}$ & 5,5 aA & $5,5 \mathrm{bA}$ & 5,8 abcA & $6,0 \mathrm{abA}$ & $5,3 \mathrm{aA}$ & 6,4 aA & 5,7 aA & $6,2 \mathrm{bA}$ & $5,8 \mathrm{bc}$ \\
\hline HR & $5,4 \mathrm{aA}$ & $5,8 \mathrm{abA}$ & $5,3 \mathrm{cA}$ & $5,7 \mathrm{bA}$ & $5,1 \mathrm{aA}$ & $5,6 \mathrm{bA}$ & 5,2 aA & $6,2 \mathrm{bA}$ & $5,5 \mathrm{~d}$ \\
\hline $\mathrm{CM}$ & $5,5 \mathrm{aA}$ & $6,0 \mathrm{abA}$ & $6,1 \mathrm{aA}$ & 5,8 abA & $5,2 \mathrm{aA}$ & $6,3 \mathrm{aA}$ & 5,8 aA & 6,4 abA & $5,9 \mathrm{~b}$ \\
\hline $\mathrm{SC}$ & 5,6 aA & 6,3 aA & $5,9 \mathrm{abA}$ & $6,3 \mathrm{aA}$ & 5,4 aA & 6,8 aA & 5,8 aA & 6,8 aA & $6,1 \mathrm{a}$ \\
\hline \multirow[t]{2}{*}{ CV (\%) } & 4,41 & 6,63 & 7,62 & 5,43 & 4,33 & 6,14 & 5,86 & 4,56 & 1,40 \\
\hline & \multicolumn{9}{|c|}{$0,15-0,30 \mathrm{~m}$} \\
\hline $\mathrm{RÇ}$ & 5,4 aA & $5,3 \mathrm{aA}$ & $5,4 \mathrm{abA}$ & 5,2 abcA & $5,4 \mathrm{aA}$ & $6,4 \mathrm{bA}$ & $5,9 \mathrm{aA}$ & 5,9 aA & $5,6 \mathrm{ab}$ \\
\hline GR & $4,8 \mathrm{aA}$ & $5,4 \mathrm{aA}$ & $5,4 \mathrm{abA}$ & $5,5 \mathrm{abA}$ & $5,4 \mathrm{aA}$ & $6,4 \mathrm{bA}$ & $5,7 \mathrm{abA}$ & $5,8 \mathrm{aA}$ & $5,6 \mathrm{ab}$ \\
\hline $\mathrm{RT}$ & $5,0 \mathrm{aA}$ & $5,2 \mathrm{aA}$ & $4,9 \mathrm{bA}$ & 5,5 abA & $5,2 \mathrm{aA}$ & $6,2 \mathrm{bA}$ & $5,7 \mathrm{abA}$ & 6,0 aA & $5,5 \mathrm{bc}$ \\
\hline $\mathrm{HC}$ & $5,3 \mathrm{aA}$ & $5,4 \mathrm{aA}$ & $5,2 \mathrm{bA}$ & 5,3 abcA & $5,3 \mathrm{aA}$ & $6,1 \mathrm{bA}$ & $5,5 \mathrm{abA}$ & $5,2 \mathrm{bA}$ & $5,4 \mathrm{bc}$ \\
\hline $\mathrm{HR}$ & $5,2 \mathrm{aA}$ & $5,5 \mathrm{aA}$ & $5,0 \mathrm{bA}$ & $4,9 \mathrm{bcA}$ & $4,8 \mathrm{aA}$ & $5,2 \mathrm{cA}$ & $5,1 \mathrm{bA}$ & $5,2 \mathrm{bA}$ & $5,1 \mathrm{c}$ \\
\hline $\mathrm{CM}$ & 5,3 aA & 5,5 aA & $5,1 \mathrm{bA}$ & $4,8 \mathrm{cA}$ & $5,1 \mathrm{aA}$ & $6,3 \mathrm{bA}$ & $5,7 \mathrm{abA}$ & $5,9 \mathrm{aA}$ & $5,5 \mathrm{bc}$ \\
\hline $\mathrm{SC}$ & 5,4 aA & 5,7 aA & 5,9 aA & $5,6 \mathrm{aA}$ & $5,4 \mathrm{aA}$ & 7,0 aA & 6,1 aA & 6,4 aA & $5,9 \mathrm{a}$ \\
\hline CV (\%) & 4,85 & 9,06 & 4,65 & 7,16 & 4,37 & 5,45 & 7,08 & 5,75 & 7,85 \\
\hline
\end{tabular}

Médias seguidas pelas mesmas letras, minúsculas na vertical e maiúsculas na horizontal, não diferem entre si pelo teste Duncan a $5 \%$. RÇ: roçadora; GR: grade; RT: enxada rotativa; HC: herbicida de pós-emergência; HR: herbicida de pré-emergência; CM: capina manual; SC: testemunha sem capina. 
compararam os tratamentos entre si. A tendência geral, representada pela média dos oito biênios, revelou que os maiores valores de $\mathrm{pH}$ são encontrados no tratamento mantido sem capina (SC), e os menores, no tratamento com herbicida de pré-emergência (HR). Quanto aos demais tratamentos (RÇ, GR, RT, HC e CM), observaram-se valores intermediários aos tratamentos SC e HR. Entre as camadas, os valores de $\mathrm{pH}$ foram mais elevados na camada superficial do solo $(0-0,15 \mathrm{~m})$.

Alcântara \& Ferreira (2000), ao estudarem o efeito dos tratamentos sobre os teores de matéria orgânica, verificaram que, tanto na camada de $0-0,15$ como na de $0,15-0,30 \mathrm{~m}$, o SC foi, entre os métodos de controle de plantas invasoras, o que promoveu, em média, o maior incremento nos teores de matéria orgânica; 0 HR, menor incremento; e os demais métodos, comportamento intermediário quanto aos teores de matéria orgânica.

O maior incremento no teor de matéria orgânica observado por Alcântara \& Ferreira (2000), no tratamento $\mathrm{SC}$, provavelmente contribuiu para elevação do $\mathrm{pH}$ em relação aos demais tratamentos (Quadro 2). Aumento de $\mathrm{pH}$ devido ao aumento no teor de matéria orgânica tem sido verificado por diferentes pesquisadores (Ismail et al., 1994; Theodoro et al., 2003). Theodoro et al. (2003), ao compararem as mudanças ocorridas nos atributos de um Latossolo Vermelho distrófico (LVd), cultivado com cafeeiro, em sistemas orgânico, em conversão e convencional, em relação ao solo sob mata nativa, verificaram aumento de pH nos sistemas orgânico e em conversão, devido às práticas de calagem, adubação orgânica e cobertura vegetal permanente no solo. Segundo os autores, o sistema de manejo orgânico caracteriza-se, desde o início, em 1995, pela adoção do manejo da lavoura, de acordo com as normas de agricultura orgânica (Brasil, 1999). Assim, foram utilizadas no manejo da lavoura adubações orgânicas (estercos de galinha, húmus de minhoca, composto à base de esterco de gado e cascas do fruto do cafeeiro e dejetos de suínos), calagem, adubação verde (Crotalaria juncea e Cajanus cajan), adubação fosfatada (termofosfato), adubações foliares com micronutrientes e calda bordalesa. O sistema em conversão caracteriza o período de transição da agricultura convencional para a agricultura orgânica, ou seja, entre 1995 e 1998 a cultura foi conduzida sob sistema de manejo convencional e, a partir de 1998, pelo sistema orgânico, conforme descrito anteriormente. Já o sistema de manejo convencional constou, desde o início de implantação da lavoura, de adubações com uréia, sulfato de amônio, superfosfato simples, cloreto de potássio, utilização de herbicidas (oxyfluorfen, glyphosate e paraquat), fungicida (epoxiconazole), adubação orgânica com casca do fruto do cafeeiro e adubações foliares com micronutrientes.

Valores mais elevados de $\mathrm{pH}$ na camada superficial do solo que em profundidade no perfil têm sido também verificados por diferentes pesquisadores (De Maria, 1999; Santos et al., 2001; Falleiro et al., 2003). Esse efeito está relacionado ao poder tamponante da matéria orgânica e, ou, ao aumento da força iônica da solução do solo, devido ao aumento nos teores de alguns nutrientes na camada superficial (Cadavid et al., 1998; Franchini et al., 1999).

Além dos efeitos anteriores, a matéria orgânica também contribui para elevação da concentração de cátions de reação básica (Iyamuremye \& Dick, 1996) e redução nos demais componentes da acidez $(\mathrm{H}+\mathrm{Al}$, $\mathrm{Al}^{3+}$ e saturação por $\mathrm{Al}^{3+}$ ) (Quadros 3, 4 e 5, respectivamente). De fato, o comportamento observado neste estudo para $\mathrm{pH}$ mostrou-se inverso para os demais componentes de acidez. Em média, o tratamento $\mathrm{HR}$ apresentou os maiores valores de acidez potencial $\left(\mathrm{H}+\mathrm{Al}\right.$ ) (Quadro 3), de $\mathrm{Al}^{3+}$ (Quadro 4) e de saturação por $\mathrm{Al}^{3+}$ (Quadro 5); o $\mathrm{SC}$, por sua vez, os menores valores para essas propriedades; e os demais tratamentos (RÇ, GR, RT, HC e CM), comportamento intermediário.

Theodoro et al. (2003) constataram ausência de $\mathrm{Al}^{3+}$ no solo nos sistemas de café orgânico e café em conversão, devido, principalmente, ao aumento do $\mathrm{pH}$, e à provável complexação do $\mathrm{Al}^{3+}$ por compostos orgânicos depositados no solo. O efeito da matéria orgânica na complexação do $\mathrm{Al}^{3+}$ e na elevação do $\mathrm{pH}$ tem sido observado na literatura (Sidiras \& Pavan, 1985; Pavan et al., 1986).

No presente estudo, a complexação do $\mathrm{Al}^{3+}$ pela matéria orgânica é, provavelmente, verificada pelos menores teores de $\mathrm{Al}^{3+}$ e menor saturação por $\mathrm{Al}^{3+}$ na camada de 0-0,15 m (Quadros 4 e 5, respectivamente), onde ocorre maior teor de matéria orgânica, conforme verificado por Alcântara \& Ferreira (2000). Por outro lado, o maior teor de $\mathrm{Al}^{3+}$ e a maior saturação por $\mathrm{Al}^{3+}$ ocorrem, exatamente, na camada onde os teores de matéria orgânica foram menores $(0,15-0,30 \mathrm{~m})$, segundo esses mesmos autores. Falleiro et al. (2003), ao compararem o efeito de sistemas de preparo de solo sobre atributos químicos e físicos de um solo, encontraram teores menores de $\mathrm{Al}^{3+}$ na camada superficial quando sob semeadura direta. Segundo esses autores, a redução nos teores de $\mathrm{Al}^{3+}$ deve-se ao incremento da força iônica da solução, em razão do aumento dos teores de $\mathrm{Ca}^{2+}, \mathrm{Mg}^{2+} \mathrm{e} \mathrm{K}^{+}$, diminuindo a atividade do $\mathrm{Al}^{3+}$ na solução; ao aumento do $\mathrm{pH}$, que afeta a especiação e solubilização do $\mathrm{Al}^{3+}$; e ao aumento do teor de matéria orgânica, que proporciona maior complexação do elemento nesta camada.

\section{Relação entre teor de matéria orgânica e componentes da acidez do solo}

Considerando apenas as médias dos anos de avaliação de cada método de controle de plantas invasoras, verificou-se que os valores de $\mathrm{pH}$ foram incrementados em resposta à elevação do teor de matéria orgânica nas camadas superficial (Figura 1a) 
Quadro 3. Acidez potencial (H + Al) do solo das camadas de 0-0,15 e 0,15-0,30 m de um Latossolo Vermelho distroférrico, em função de métodos de controle de plantas invasoras na cultura do cafeeiro

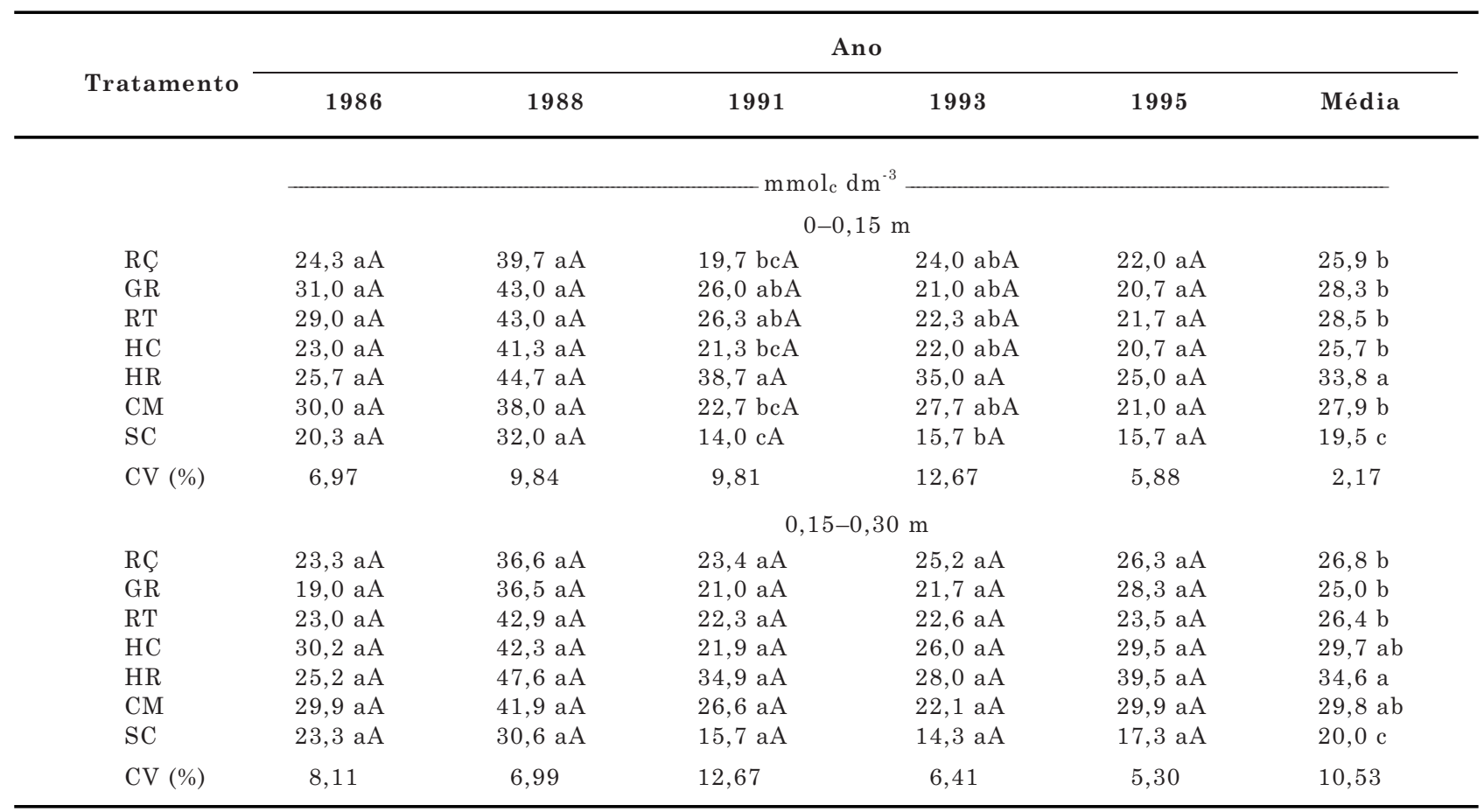

Médias seguidas pelas mesmas letras, minúsculas na vertical e maiúsculas na horizontal, não diferem entre si pelo teste Duncan a $5 \%$. RÇ: roçadora; GR: grade; RT: enxada rotativa; HC: herbicida de pós-emergência; HR: herbicida de pré-emergência; CM: capina manual; SC: testemunha sem capina.

Quadro 4. Teor de $\mathrm{Al}^{3+}$ nas camadas de 0,0-0,15 e 0,15-0,30 m de um Latossolo Vermelho distroférrico, em função dos métodos de controle de plantas invasoras na cultura do cafeeiro

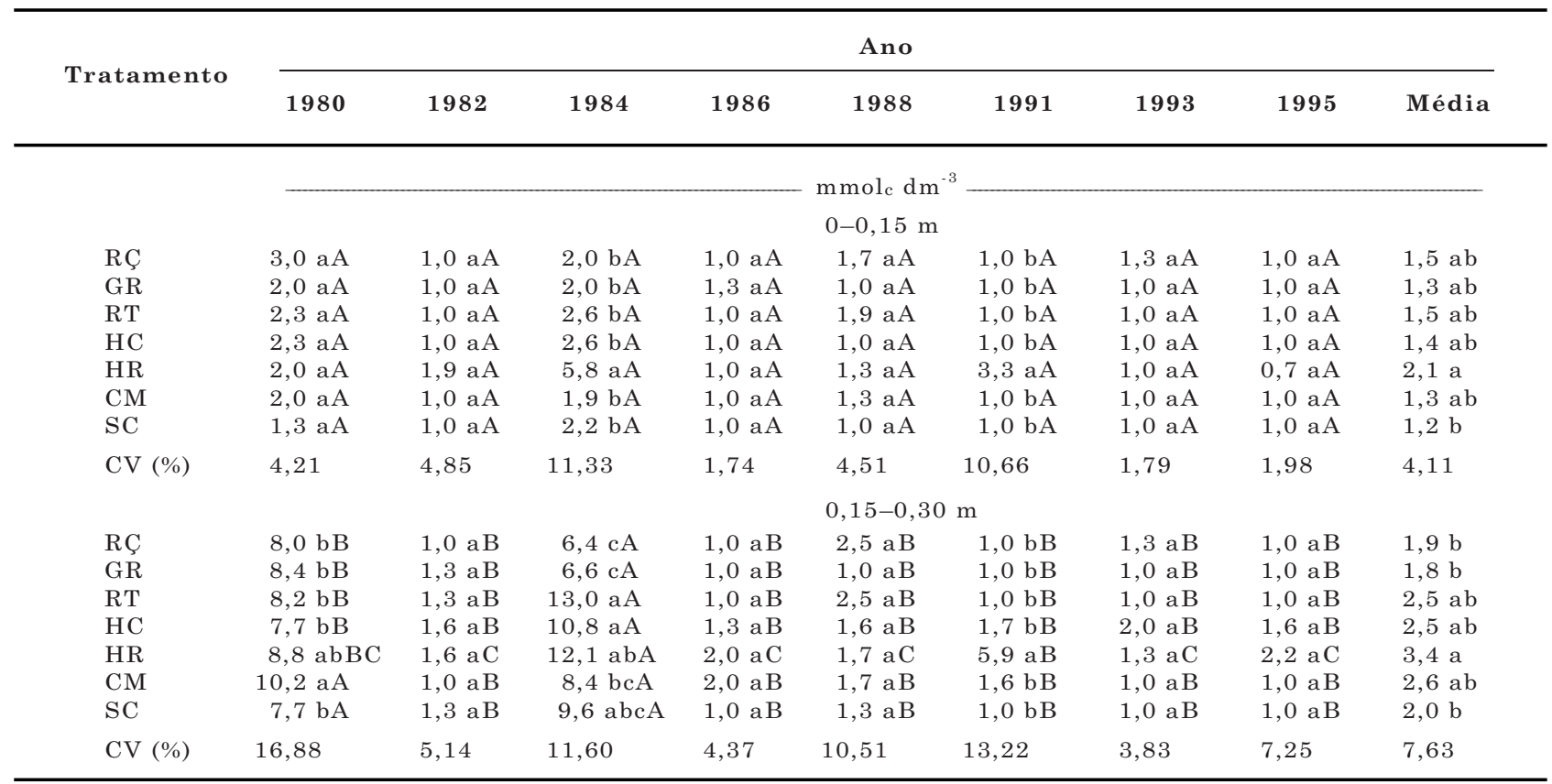

Médias seguidas pelas mesmas letras, minúsculas na vertical e maiúsculas na horizontal, não diferem entre si pelo teste Duncan a $5 \%$. RÇ: roçadora; GR: grade; RT: enxada rotativa; HC: herbicida de pós-emergência; HR: herbicida de pré-emergência; CM: capina manual; SC: testemunha sem capina. 
Quadro 5. Saturação por $\mathrm{Al}^{3+}$ do solo das camadas de 0-0,15 e 0,15-0,30 m de um Latossolo Vermelho distroférrico, em função de métodos de controle de plantas invasoras na cultura do cafeeiro

\begin{tabular}{|c|c|c|c|c|c|c|}
\hline \multirow{2}{*}{ Tratamento } & \multicolumn{6}{|c|}{ Ano } \\
\hline & 1986 & 1988 & 1991 & 1993 & 1995 & Média \\
\hline & \multicolumn{6}{|c|}{$0-0,15 \mathrm{~m}$} \\
\hline $\mathrm{RÇ}$ & $2,00 \mathrm{bB}$ & $6,00 \mathrm{bcA}$ & $2,00 \mathrm{bB}$ & $4,00 \mathrm{abB}$ & $1,50 \mathrm{bcB}$ & $3,10 \mathrm{~b}$ \\
\hline GR & $4,67 \mathrm{aA}$ & $4,33 \mathrm{cdA}$ & $2,00 \mathrm{bB}$ & $2,00 \mathrm{bB}$ & $1,33 \mathrm{bcB}$ & $2,87 \mathrm{bc}$ \\
\hline $\mathrm{RT}$ & $2,67 \mathrm{abAB}$ & $4,67 \mathrm{cdA}$ & $2,17 \mathrm{bB}$ & $2,00 \mathrm{bB}$ & $2,00 \mathrm{abB}$ & $2,70 \mathrm{bc}$ \\
\hline $\mathrm{HC}$ & $2,33 \mathrm{abB}$ & $7,00 \mathrm{bA}$ & $1,83 \mathrm{bB}$ & $1,67 \mathrm{bB}$ & $1,33 \mathrm{bcB}$ & $2,83 \mathrm{bc}$ \\
\hline HR & $2,67 \mathrm{abC}$ & $13,17 \mathrm{bcA}$ & 3,00 aBC & $6,67 \mathrm{aB}$ & $2,50 \mathrm{aC}$ & $5,60 \mathrm{a}$ \\
\hline $\mathrm{CM}$ & $2,67 \mathrm{abB}$ & $6,00 \mathrm{bcA}$ & $2,00 \mathrm{bB}$ & $1,33 \mathrm{bB}$ & $1,50 \mathrm{bcB}$ & $2,70 \mathrm{bc}$ \\
\hline $\mathrm{SC}$ & $1,67 \mathrm{bB}$ & $4,00 \mathrm{dA}$ & $1,00 \mathrm{cB}$ & $1,00 \mathrm{bB}$ & $1,00 \mathrm{cB}$ & $1,73 \mathrm{c}$ \\
\hline \multirow[t]{2}{*}{ CV (\%) } & 18,91 & 4,60 & 2,70 & 3,02 & 3,01 & 18,26 \\
\hline & \multicolumn{6}{|c|}{$0,15-0,30 \mathrm{~m}$} \\
\hline $\mathrm{RÇ}$ & $6,28 \mathrm{aA}$ & 3,16 aA & $3,28 \mathrm{aA}$ & $6,50 \mathrm{aA}$ & $3,41 \mathrm{aA}$ & $4,44 \mathrm{~b}$ \\
\hline GR & $5,91 \mathrm{aA}$ & $3,70 \mathrm{aA}$ & $2,00 \mathrm{aA}$ & $4,32 \mathrm{aA}$ & $3,00 \mathrm{aA}$ & $3,67 \mathrm{~b}$ \\
\hline $\mathrm{RT}$ & $5,60 \mathrm{aA}$ & $4,12 \mathrm{aA}$ & $2,00 \mathrm{aA}$ & 3,32 aA & $2,64 \mathrm{aA}$ & $3,43 \mathrm{~b}$ \\
\hline $\mathrm{HC}$ & 7,48 aA & $6,17 \mathrm{aA}$ & $1,63 \mathrm{aA}$ & $4,91 \mathrm{aA}$ & 5,22 aA & $4,85 \mathrm{~b}$ \\
\hline HR & $12,03 \mathrm{aA}$ & 8,16 aA & $6,03 \mathrm{aA}$ & $7,25 \mathrm{aA}$ & $15,34 \mathrm{aA}$ & $9,51 \mathrm{a}$ \\
\hline $\mathrm{CM}$ & $15,69 \mathrm{aA}$ & $6,01 \mathrm{aA}$ & $1,30 \mathrm{aA}$ & $3,22 \mathrm{aA}$ & $4,00 \mathrm{aA}$ & $5,20 \mathrm{~b}$ \\
\hline $\mathrm{SC}$ & $4,65 \mathrm{aA}$ & $3,34 \mathrm{aA}$ & $1,00 \mathrm{aA}$ & $1,63 \mathrm{aA}$ & $1,83 \mathrm{aA}$ & $2,32 \mathrm{~b}$ \\
\hline CV (\%) & 27,06 & 31,28 & 33,39 & 30,71 & 33,29 & 37,02 \\
\hline
\end{tabular}

Médias seguidas pelas mesmas letras, minúsculas na vertical e maiúsculas na horizontal, não diferem entre si pelo teste Duncan a $5 \%$. RÇ: roçadora; GR: grade; RT: enxada rotativa; HC: herbicida de pós-emergência; HR: herbicida de pré-emergência; CM: capina manual; SC: testemunha sem capina.

$(\mathrm{p}<0,01)$ e subsuperficial (Figura 1b) $(\mathrm{p}<0,05)$ enquanto $\mathrm{H}+\mathrm{Al}$ apresentou comportamento inverso, ou seja, decréscimo com o aumento do nível de matéria orgânica, tanto na camada superficial (Figura 1c) $(\mathrm{p}<0,01)$ como na subsuperficial (Figura 1d) $(\mathrm{p}<0,05)$. O teor de $\mathrm{Al}^{3+}$ e a saturação por $\mathrm{Al}^{3+}$ nas camadas superficial (Figura 1e,g, respectivamente) e subsuperficial (Figura 1f,h, respectivamente) não apresentaram, em média, resposta significativa ao aumento do teor de matéria orgânica $(p>0,05)$. Entretanto, o $\mathrm{Al}^{3+}$ e a saturação por $\mathrm{Al}^{3+}$ tenderam a apresentar comportamento inverso ao dos incrementos do teor de matéria orgânica.

A resposta do $\mathrm{pH}$ aos diferentes métodos de controle de plantas invasoras foi determinada em certa extensão pelo incremento nos teores de matéria orgânica e pela redução nos teores de $\mathrm{H}+\mathrm{Al}, \mathrm{Al}^{3+} \mathrm{e}$ saturação por $\mathrm{Al}^{3+}$, uma vez que essas variáveis apresentam correlação com o pH do solo (Quadro 6). O efeito da matéria orgânica na diminuição da acidez do solo tem sido observado na literatura (Sidiras \& Pavan, 1985; Ismail et al., 1994; Theodoro et al., 2003).

\section{CONCLUSÕES}

1. Os métodos de controle de plantas invasoras alteraram, de forma diferenciada, os componentes da acidez do solo.

2. O tratamento sem capina (SC) promoveu o maior incremento nos valores de $\mathrm{pH}$ e redução da acidez potencial, do teor de $\mathrm{Al}^{3+}$ e da saturação por $\mathrm{Al}^{3+}$. 


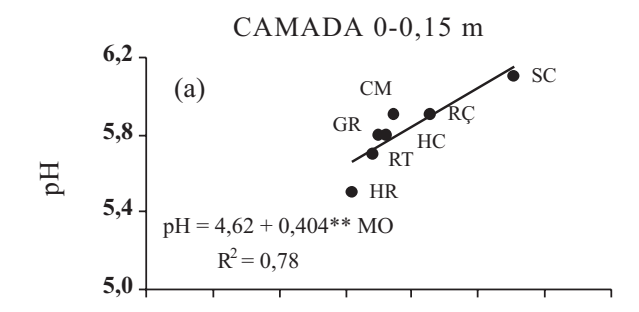

CAMADA $0,15-0,30 \mathrm{~m}$
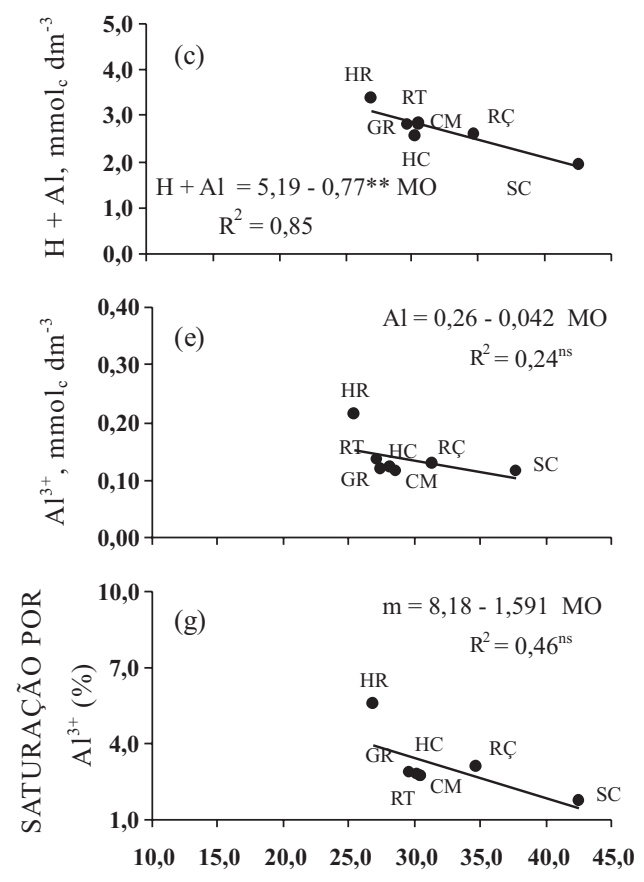

$\mathrm{MO}, \mathrm{g} \mathrm{kg}^{-1}$
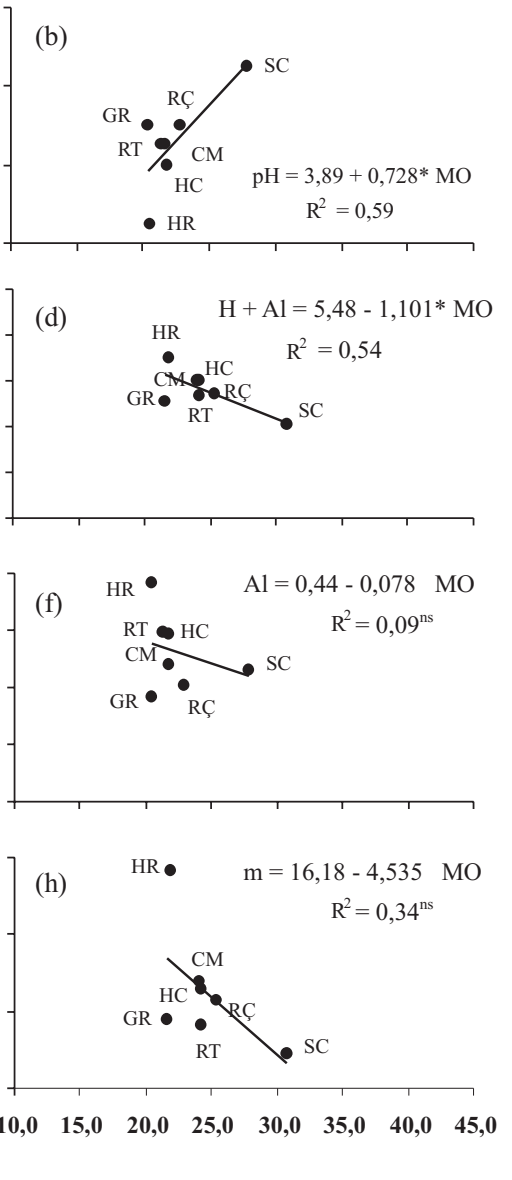

Figura 1. Regressões entre teor de matéria orgânica e $\mathrm{pH}, \mathrm{H}+\mathrm{Al}, \mathrm{Al}^{3+}$ trocável e saturação por $\mathrm{Al}^{3+}$ para as camadas de 0-0,15 e 0,15-0,30 m de um Latossolo Vermelho distroférrico, considerando diferentes métodos de controle de plantas invasoras (RÇ: roçadora; GR: grade; RT: enxada rotativa; HC: herbicida de pós-emergência; HR: herbicida de pré-emergência, CM: capina manual; SC: testemunha sem capina) na cultura do cafeeiro.

Quadro 6. Correlação entre pH e matéria orgânica, acidez potencial $(H+A 1)$, alumínio $\left(\mathbf{A 1}^{3+}\right)$ e saturação por $\mathrm{A1}^{3+}$ nas camadas de 0-0,15 e de 0,15-0,30 m de um Latossolo Vermelho distroférrico, cultivado com cafeeiro sob diferentes métodos de controle de plantas invasoras

\begin{tabular}{|c|c|c|c|c|c|c|c|c|}
\hline & \multicolumn{4}{|c|}{ Camada de $0-0,15 \mathrm{~m}$} & \multicolumn{4}{|c|}{ Camada de $0,15-0,30 \mathrm{~m}$} \\
\hline & MO & $\mathbf{H}+\mathbf{A l}$ & $\mathrm{Al}^{3+}$ & m & MO & $\mathbf{H}+\mathbf{A l}$ & $\mathrm{Al}^{3+}$ & $\mathbf{m}$ \\
\hline $\mathrm{pH}$ & $0,88^{* *}$ & $-0,93 * *$ & $-0,81^{* *}$ & $0,87 * *$ & $0,75^{*}$ & $-0,97 * *$ & $0,78^{*}$ & $-0,90 * *$ \\
\hline
\end{tabular}

* e **: significativo a 5 e $1 \%$, respectivamente.

3. O tratamento com herbicida de pré-emergência (HR) destacou-se, negativamente, dentre os métodos de controle de plantas invasoras, por elevar o teor de $\mathrm{Al}^{3+}$, a saturação por $\mathrm{Al}^{3+}$ e a acidez potencial e diminuir o $\mathrm{pH}$ do solo.

\section{LITERATURA CITADA}

AASE, J.K. \& PIKUL Jr., J.L. Crop and soil response to longterm tillage practices in the Norther Great Plains. Agron. J., 87:652-656, 1995. 
ALCÂNTARA, E.N. \& FERREIRA, M.M. Efeitos de métodos de controle de plantas daninhas na cultura do cafeeiro (Coffea arábica L.) sobre a qualidade física do solo. R. Bras. Ci. Solo, 24:711-721, 2000.

BAYER, C. \& BERTOL, I. Características químicas de um Cambissolo Húmico afetadas por sistemas de preparo, com ênfase à matéria orgânica. R. Bras. Ci. Solo, 23:687694, 1999.

BAYER, C. \& MIELNICZUK J. Características químicas do solo afetadas por métodos de preparo e sistemas de cultura. R. Bras. Ci. Solo, 21:105-112, 1997.

BRASIL. Ministério da Agricultura e do Abastecimento. Instrução normativa, $\mathrm{n}^{\circ} 7$, de 17 de maio de 1999. Dispõe sobre a produção de produtos orgânicos vegetais e animais; LEX, 63:2465-2476, 1999. (Coletânea de Legislação e Jurisprudência: legislação federal e marginalia).

BURLE, M.L.; MIELNICZUK, J. \& FOCCHI, S. Effect of cropping systems on soil chemical characteristics, with emphasis on soil acidification. Plant Soil, 190:309-316, 1997.

CADAVID, L.F.; EL-SHARKAWY, M.A.; ACOSTA, A. \& SÁNCHEZ, T. Long-term effects of mulch, fertilization and tillage on cassava grown in sandy soils in Northern Colombia. Field Crops Res., 57:45-56, 1998.

COMISSÃO DE FERTILIZANTES DO SOLO DO ESTADO DE MINAS GERAIS - CFSEMG. Recomendações para o uso de corretivos e fertilizantes em Minas Gerais, $4^{\text {a }}$ aproximação. Lavras, 1989. 176p.

DE MARIA, I.C.; NnABUDE, P.C. \& CASTRO, O.M. Longterm tillage and crop rotation effects on soil chemical proprieties of a Rholic Ferrasol in Southern Brasil. Soil Till. Res., 51:71-79, 1999.

EMPRESA BRASILEIRA DE PESQUISA AGROPECUÁRIA EMBRAPA. Serviço Nacional de Levantamento e Conservação de Solos. Manual de métodos de análises de solo. Rio de Janeiro, 1979. 240p.

FALLEIRO, R.M.; SOUZA, C.M.; SILVA, C.S.W.; SEDIYAMA, C.S.; SILVA, A.A. \& FAGUNDES, J.L. Influência dos sistemas de preparo nas propriedades químicas e físicas do solo. R. Bras. Ci. Solo, 27:1097-1104, 2003.
FRANCHINI, J.C.; MALAVOLTA, E.; MIYAZAWA, M. \& PAVAN, M.A. Alterações químicas em solos ácidos após a aplicação de resíduos vegetais. R. Bras. Ci. Solo, 23:533$542,1999$.

ISMAIL, I.; BLEVINS, R.L. \& FRYE, W.W. Long term notillage on soil properties and continuous corn yields. Soil Sci. Soc. Am. J., 58:193-198, 1994.

IYAMUREMYE, F. \& DICK, R.P. Organic amendments and phosphorus sorption by soils. Adv. Agron., 56:139-185, 1996.

PAVAN, M.A.; CARAMORI, P.H.; ANDROCIOLI FILHO, A. \& SCHOLZ, M.F. Manejo da cobertura do solo para formação e produção de uma lavoura cafeeira. I. Influência na fertilidade do solo. Pesq. Agropec. Bras., 21:187-192, 1986.

REICOSKY, D.C.; KEMPER, W.D.; LANGDALE., G.W.; DOUGLAS Jr., C.L. \& RASMUNSSEN, P.E. Soil organic matter changes resulting from tillage and biomass production. J. Soil Water Conserv., 50:253-261, 1995.

SANTOS, A.C.; SILVA, I.F.; LIMA, J.R.S.; ANDRADE, A.P. \& CAVALCANTE, V.R. Gramíneas e leguminosas na recuperação de áreas degradadas: efeito nas características químicas de solo. R. Bras. Ci. Solo, 25:10631071, 2001.

SANTOS, H.P. \& TOMM, G.O. Estudo da fertilidade do solo sob quatro sistemas de rotação de culturas envolvendo trigo em plantio direto. R. Bras. Ci. Solo, 20:407-414, 1996.

SIDIRAS, N. \& PAVAN, M.A. Influência do sistema de manejo do solo no seu nível de fertilidade. R. Bras. Ci. Solo, 9:249$254,1985$.

SILVA, M.S.L. \& RIBEIRO, M.R. Influência do cultivo contínuo da cana-de-açúcar nas propriedades químicas de solos argilosos. Pesq. Agropec. Bras., 30:389-394, 1995.

THEODORO, V.C.A.; ALVARENGA, M.I.N.; GUIMARÃES, R.J. \& SOUZA, C.A.S. Alterações químicas em solo submetido a diferentes formas de manejo do cafeeiro. R. Bras. Ci. Solo, 27:1039-1047, 2003.

VETTORI, L. Métodos de análises de solos. Rio de Janeiro, Ministério da Agricultura, 1969. 24p. (Boletim Técnico, 7) 